\title{
Duftverarbeitung im Antennallobus der Honigbiene Apis mellifera
}

\author{
Silke Sachse und C. Giovanni Galizia
}

\begin{abstract}
Zusammenfassung
Gehirne bestehen aus komplexen Netzwerken von Neuronen, die Informationen - in Form von neuronaler Aktivität - verarbeiten, optimieren und selektieren. Um grundsätzliche Mechanismen von Informationsverarbeitung zu verstehen, haben wir uns mit dem Geruchssinn beschäftigt. Hierzu haben wir eine klar abgegrenzte Struktur im Insektengehirn, den olfaktorischen Antennallobus, der das Riechhirn der Insekten darstellt, untersucht. Durch gleichzeitige Visualisierung der Aktivität des Eingangs und des Ausgangs haben wir die Verarbeitung der olfaktorischen Information durch das neuronale Netzwerk des Antennallobus analysiert. Mittels pharmakologischer Methoden haben wir Teile dieses Netzwerkes ausgeschaltet. Die Ergebnisse zeigen, dass die Duftrepräsentation durch mehrere parallele Netzwerke moduliert wird, die unterschiedliche Aspekte der Duftverarbeitung optimieren. Es konnten mindestens zwei Funktionen voneinander getrennt werden: ein globales Netzwerk, das die Gesamtaktivität im Optimum hält, und ein selektives Netzwerk, dessen Verschaltungen die Repräsentation verschiedener Düfte kontrastverstärkt.
\end{abstract}

\begin{abstract}
Odor processing in the honeybee antennal lobe.

Brains consist of complex neural networks which process, optimize and select information in form of neural activity. In order to understand basic mechanisms of information processing, we studied the sense of smell. We analyzed a well defined structure in the insect brain, the antennal lobe, which represents the olfactory bulb of insects. By simultaneously visualizing activity in the input and the output, we analyzed the processing of olfactory information by the antennal lobe neural network. Using pharmacological tools we selectively blocked parts of the network. Our results show that olfactory representation is modulated by several parallel networks, which optimize different aspects of olfactory coding. At least two functions could be isolated: a global network, likely to keep the overall activity in an optimal range, and a selective network. Individual connections of the latter contrast enhance the representation of different odors.
\end{abstract}

Key words: honeybee; calcium imaging; olfactory coding; antennal lobe; olfactory glomeruli

\section{Einleitung}

Über unsere fünf Sinnesorgane nehmen wir Informationen aus der Außenwelt wahr. Unser Gehirn verarbeitet diese Sinneseindrücke mittels komplexer neuronaler Verschaltungen und setzt sie zu einem sinnvollen Ganzen zusammen. Um grundlegende neuronale Verarbeitungsmechanismen zu verstehen, haben wir uns mit einer Sinnesmodalität, dem Geruchssinn, beschäftigt. Alle Organismen können auf chemische Substanzen in ihrer Umwelt reagieren. Diese Fähigkeit reicht von einfachen Einzellern, wie z.B. Bakterien und Protisten, bis hin zu komplexen Vielzellern, wie Mollusken, Arthropoden und Säugetieren. Das Erkennen von chemischen Substanzen, wie z.B. Düften, kann einen sehr spezifischen Informationsgehalt über Nahrung, Gefahr, Partnerfindung und Territorialverhal- ten liefern. Der Geruchssinn muss mit einer enormen Vielfalt an diversen Düften hantieren, welche aus kleinen, flüchtigen, meist organischen Molekülen bestehen. Die Anzahl verschiedener Duftmoleküle wurde für das menschliche Geruchssystem auf ca. 400.000 geschätzt (Mori und Yoshihara 1995), wobei jedes einzelne Molekül generell eine einzigartige Geruchsempfindung hervorruft. Schon kleinste Veränderungen in der Molekülstruktur können zu einer komplett anderen Geruchswahrnehmung führen. Die außerordentliche Fähigkeit des Geruchssystems, die enorme Anzahl an Duftmolekülen zu erkennen und zu unterscheiden, wird nur vom Immunsystem übertroffen, welches eine undefinierbare Anzahl an Antigenmolekülen bekämpfen kann. Wie vermag das Geruchssystem diese enorme Duftmolekülanzahl in eine Geruchsempfindung umzuwandeln?
In jüngster Zeit hat sich unser Verständnis der neuronalen Mechanismen, die der Duftverarbeitung im Gehirn zugrunde liegen, stark erweitert. Duftmoleküle werden über olfaktorische Rezeptorneurone wahrgenommen, die sich z.B. bei Menschen in der Nasenschleimhaut und im Gegensatz dazu bei Insekten auf den Antennen befinden. Diese Neurone exprimieren jeweils einen olfaktorischen Rezeptortyp, welcher mit dem Duftmolekül interagiert. Die Gene für diese Rezeptoren wurden in verschiedenen Tierarten identifiziert und sequenziert. Die Zahl der Rezeptorgene ist in verschiedenen Tierarten unterschiedlich und reicht von ca. 60 bei der Fruchtfliege (Review: Vosshall 2000), über 350 beim Menschen (Review: Young und Trask 2002), bis zu 1000 bei Mäusen (Review: Mombaerts 1999). Wie können mit so wenigen Rezeptortypen so viele Duftsubstanzen erkannt werden? Jedes Rezeptorprotein interagiert nicht nur mit einem, sondern mit vielen Duftmolekülen (z.B. Sicard und Holley 1984). Auf diese Weise hat das Geruchssystem eine Strategie entwickelt, die seine Kodierungsfähigkeit exponentiell vergrößert. Damit das Gehirn die verschiedenen Geruchsmoleküle auch unterscheiden kann, sollte es kleinste Unterschiede in den Aktivitätsmustern der Rezeptorneurone erkennen können. Dazu muss die Duftinformation der Rezeptorneurone verschärft und kontrastverstärkt werden. Um diesen Mechanismus der Duftverarbeitung im Gehirn zu verstehen, haben wir die Duftrepräsentation auf zwei verschiedenen Verarbeitungsebenen im Gehirn gemessen und miteinander verglichen.

\section{Die Architektur des Geruchssystems der Honigbiene}

Die Honigbiene Apis mellifera (Abbildung 1A) eignet sich sehr gut als ein Modellorganismus, um die Verarbeitung von Düften im Gehirn zu verstehen. Die Biene ist hochgradig davon abhängig, Düfte zu erkennen, zu klassifizieren und zu lernen. Sie wird vom ersten Lebensabschnitt im Stock bis zur Tätigkeit im Freien als Sammlerin durch ihren Geruchssinn geleitet (Frisch 1993). Bienen sind soziale Insekten und kommunizieren mit ihren Artgenossen auch über selbst erzeugte Signaldüfte, die Pheromone. Als Sammlerinnen orientieren sie sich an der Farbe, der Form und dem bezeichnenden Duft einer Blume, um später die nektarreichen Blüten auf einer Wiese wieder zu finden. Das Geruchssystem der Biene ist in Abbildung 1B dargestellt. Die 60.000 olfaktorischen Rezeptorneurone sitzen auf der 


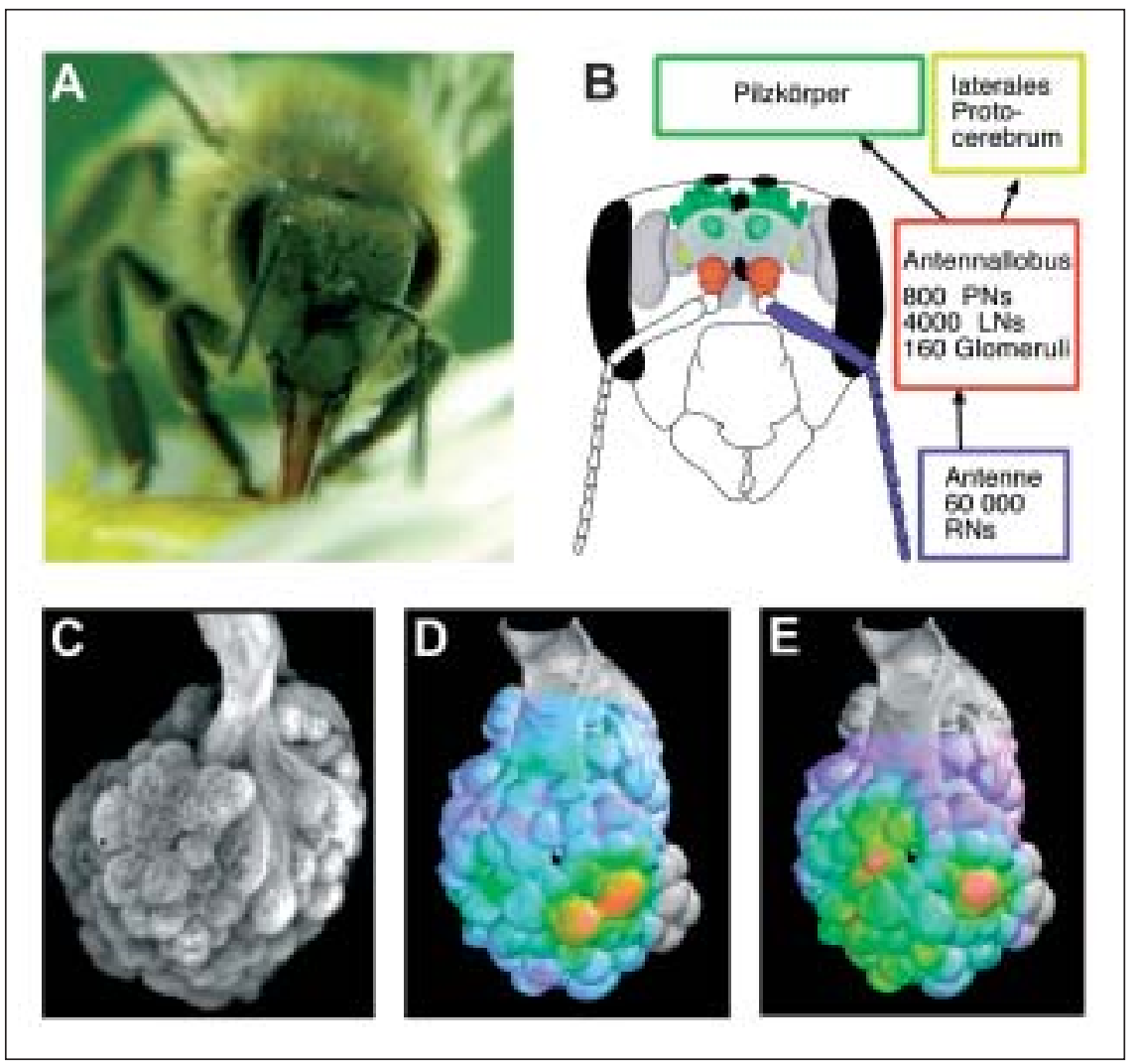

Abb. 1: Das olfaktorische System der Honigbiene. (A) Die Honigbiene Apis mellifera sitzt nektarsaugend auf einer Blüte. (B) Schematischer Aufbau der olfaktorischen Bahn der Biene. Die olfaktorischen Rezeptorneurone (RNs) sitzen auf der Antenne (blau) und innervieren über den Antennalnerv den Antennallobus (rot), in dem die primäre Verschaltung zwischen Rezeptorneuronen, lokalen Interneuronen (LNs) und Projektionsneuronen (PNs) stattfindet. Letztere projizieren in Zentren höherer Verarbeitung, wie den Pilzkörper (grün) und das laterale Protocerebrum (gelb). (C) Konfokale Aufnahme des Antennallobus. Der Antennalnerv befindet sich oben, die kugeligen Strukturen sind die Glomeruli. (D und E) Zwei Calcium Imaging Aktivitätsbilder während der Duftgabe von Nonanol bzw. Nelkenöl auf schematisierten Antennalloben. Die Farbkodierung spiegelt die neuronale Aktivität wider. Beide Düfte erzeugen kombinatorische Muster aktivierter Glomeruli.
Antenne (Esslen und Kaissling 1976) und innervieren über vier antennale Nerventrakte (Suzuki 1975) die erste Schaltstation des Bienengehirns, den Antennenlappen oder Antennallobus (AL). Der AL entspricht sowohl strukturell als auch funktionell dem Riechhirn der Wirbeltiere, dem Bulbus olfaktorius (Hildebrand und Shepherd 1997). Der AL besteht aus ca. 160 kugeligen Strukturen, den olfaktorischen Glomeruli, die strukturelle Untereinheiten darstellen und einschichtig das äußere Volumen des ALs formen (Flanagan und Mercer 1989; Abbildung 1C). Die Glomeruli sind die funktionellen Einheiten der olfaktorischen Informationsverarbeitung. Sie bestehen aus einem dichten Geflecht von Axonen und Dendriten dreier Neuronentypen, die sich hier synaptisch miteinander verschalten. Die Rezeptorneurone konvergieren auf ca. $4.000 \mathrm{Neu}-$ rone (Witthöft 1967), die aufgrund ihrer ausschließlichen Verzweigungen innerhalb des ALs als lokale Interneurone bezeichnet werden. Den dritten Neuronentyp stellen die Projektionsneurone dar (ca. 800; Bicker et al. 1993; Hammer 1997), welche jeweils mit Rezeptorneuronen und lokalen Interneuronen synaptisch interagieren. Die Projektionsneurone senden über drei verschiedene Nerventrakte die olfaktorische Information vom AL zu höheren Verarbeitungsstrukturen, wie das laterale Protocerebrum und den Pilzköper (Mobbs 1982). Die Zellkörper von Inter- und Projektionsneuronen liegen peripher am AL angelagert (Arnold et al. 1985). Jeder einzelne Glomerulus besitzt eine charakteristische Größe und Lage, was seine Identifizierung mittels eines morphologischen Atlas ermöglicht (Flanagan und Mercer 1989; Galizia et al. 1999a). 


\section{Repräsentation der Düfte im Antennallobus}

Wie werden Düfte im AL neuronal kodiert? Die physiologische Antwort der Glomeruli im AL kann mittels der Calcium Imaging Methode visualisiert werden (Review: Galizia und Menzel 2001). Die Methode beruht darauf, dass das Gehirn mit einem kalziumsensitiven Farbstoff gefärbt wird, welcher seine Fluoreszenz entsprechend der Kalziumkonzentration verändert. Kalzium ist ein sekundärer Botenstoff in einer Reihe von Signalkaskaden und spielt bei der synaptischen Übertragung eine entscheidende Rolle. Demzufolge eignet sich Kalzium sehr gut als Indikator für neuronale Aktivität. Das Imaging ermöglicht, dass die neuronale Aktivität im Gehirn mittels einer hochsensitiven Kamera gleichzeitig an verschiedenen Orten gemessen werden kann. Es eröffnet sich also die raum-zeitliche Aktivität der Neurone, die an der Verarbeitung eines Sinneseindrucks beteiligt sind. Man kann im wahrsten Sinne des Wortes dem Gehirn beim ,denken' zusehen. Wenn man nun die Antennen einer Biene mit einem Duft stimuliert und simultan die Kalziumveränderungen im AL misst, so sieht man, dass jeder Duft ein Muster unterschiedlich aktiver Glomeruli hervorruft (Joerges et al. 1997; Galizia et al. 1999b; Sachse et al. 1999). Abbildung $1 \mathrm{D}$ und $1 \mathrm{E}$ zeigen die Aktivitätsmuster im AL zweier Düfte. Die physiologischen Kalziumantworten wurden in Falschfarben kodiert. Diese Farbkodierung spiegelt die intrazelluläre Kalziumzunahme und damit die neuronale Aktivität wider. Die Farben von gelb bis rot stehen für starke, die Farben wie grün und blau für eine geringe bis hin zu gar keiner Erhöhung der Kalziumkonzentration. Die beiden Düfte Nonanol, ein Alkohol (Abbildung 1D), und Nelkenöl, ein komplexes Duftgemisch (Abbildung 1E), rufen charakteristische räumliche Aktivitätsmuster hervor. Nonanol aktiviert zwei benachbarte Glomeruli. Nelke hingegen ruft eine starke Aktivität in einem mit dem Nonanolmuster gemeinsamen Glomerulus und in zwei anderen Glomeruli hervor. Diese Aktivitätsmuster sind Beispiele dafür, dass Düfte als kombinatorische $\mathrm{Mu}$ ster aktivierter Glomeruli repräsentiert werden: Ein Duft ruft generell Aktivität in mehreren Glomeruli hervor, wobei ein Glomerulus an dem Muster verschiedener Düfte beteiligt sein kann. Besonders interessant ist, dass die Repräsentation von Düften zwischen den Individuen gleich und damit genetisch determiniert ist (Galizia et al. 1999b). Die Antwortstärke einzelner Glomeruli ist

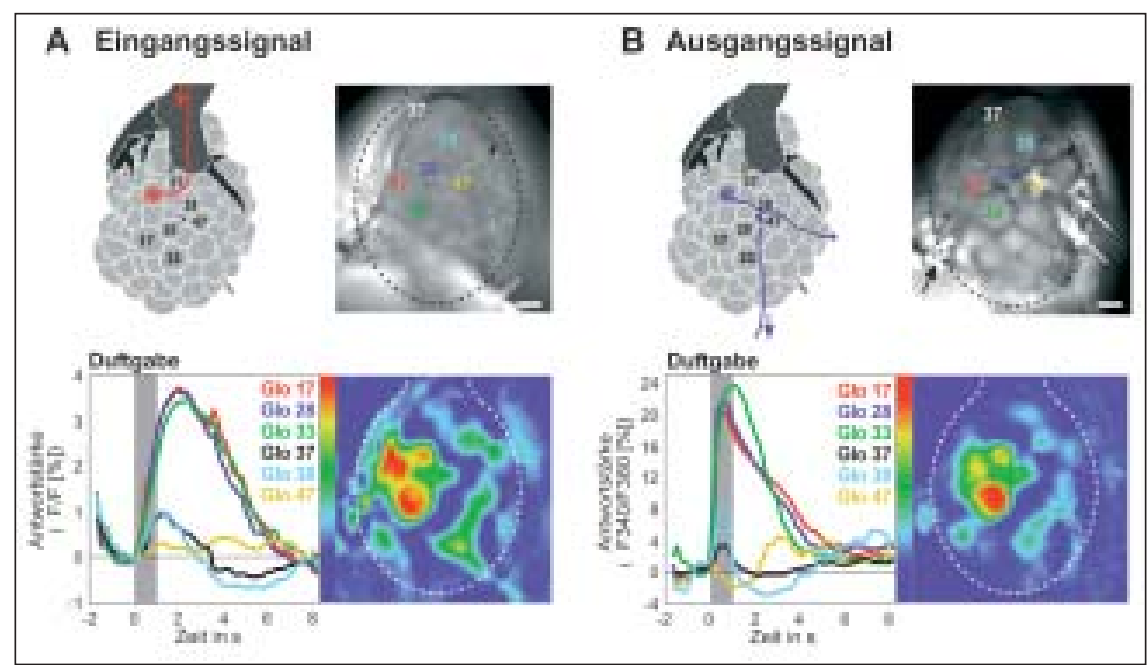

Abb. 2: Simultane Messung des Rezeptorneuron-Einganges zum AL und ProjektionsneuronAusganges. (A und B) Oben: Schematische und morphologische Darstellung des ALs. Rezeptorneurone senden ihre Axone über den Antennalnerv in den AL und innervieren einzelne Glomeruli (rotes Neuron), wobei Projektionsneurone die Duftinformation in den Glomeruli abgreifen und sie in höhere verarbeitende Strukturen senden (blaues Neuron; Zellkörper befindet sich peripher am AL). Die morphologischen Bilder repräsentieren den AL nach erfolgter Badapplikation mit Calcium Green-AM (A) und selektiver Anfärbung der Projektionsneurone mit Fura-dextran (Zellkörper der Projektionsneurone sind mit Pfeilen markiert). Einzelne Glomeruli sind benannt. Unten: Zeitliche und räumliche Antwortmuster während einer Duftstimulation (Mischung aus Hexanol, Oktanol und Nonanol) der oben identifizierten Glomeruli, welche simultan auf der Eingangsebene als auch im Ausgang gemessen wurden. Die Duftstimulation ist im Zeitverlauf als grauer Balken dargestellt. Die Zeitverläufe zeigen den Aktivitätsverlauf in den identifizierten Glomeruli.

jedoch erfahrungsabhängig und kann durch Lernen verändert werden (Faber et al. 1999).

Um nun zu untersuchen, wie der AL die Duftinformation der Rezeptorneurone verarbeitet, haben wir simultan den Eingang und den Ausgang gemessen (Sachse und Galizia 2002a). Hierzu mussten beide Verarbeitungsstufen selektiv voneinander markiert werden. Für die Messung der Rezeptorneurone wurde ein kalziumsensitiver Farbstoff (Calcium Green-AM) als Badapplikation zum Gehirn gegeben, dessen Signale dem Rezeptorneuroneingang zum Antennallobus entsprechen (Abbildung 2A). Mit einem zweiten kalziumsensitiven Farbstoff (Fura-dextran) wurden die Projektionsneurone gefärbt. Hierzu wurden Kristalle des Farbstoffs in die Axonbündel der Projektionsneurone vom AL zum Pilzkörper injiziert (Abbildung 2B). Da die beiden Farbstoffe bei verschiedenen Wellenlängen fluoreszieren, konnten sie unabhängig voneinander gemessen werden. Eine Duftstimulation führte sowohl auf der Eingangs- als auch auf der Ausgangsebene zu einem ähnlichen duftspezifischen Muster aktivierter Glomeruli. Vergleicht man jedoch die Zeitverläufe einzelner Glomeruli der beiden Verarbeitungsstufen, so sind markante Unterschiede erkennbar. Glomerulus 47 (gelber Zeitverlauf in Abbildung 2) zeigt auf der Projektionsneuronebene eine Abnahme der Kalziumkonzentration während der Duftgabe, d.h. eine inhibitorische Antwort, obwohl dieser Glomerulus ein schwaches, jedoch positives Eingangssignal erhält. Inhibitorische Antworten wurden bisher nie auf der Rezeptorebene beobachtet und scheinen damit eine spezifische Eigenschaft der Projektionsneurone zu sein, welche von inhibitorischen synaptischen Verschaltungen im AL verursacht werden. Diese Beobachtung stimmt mit elektrophysiologischen Ableitungen an Projektionsneuronen in der Honigbiene überein (Abel et al. 2001; Müller et al. 2002). Ein weiterer Unterschied der beiden Verarbeitungsstufen ist in den Erregungsverhältnissen der drei stark aktiven Glomeruli 17, 28 und 33 (rote, blaue und grüne Zeitverläufe) zu finden: Auf der Eingangsseite (Abbildung 2A) sind diese drei Glomeruli gleich stark aktiviert, während im Ausgangssignal Glomerulus 33 der stärkste ist. Generell ist die Anzahl aktiver Glomeruli im Ausgangssignal (Abbildung 2B ) reduziert. Abbildung 3 zeigt über die Tiere gemittelte und in einem standardisierten AL dargestellte Duftantwortmuster. Die glomeruläre Antwortstärke wurde hierzu in fünf gleich große Kategorien eingeteilt und farblich kodiert. Inhibitorische Antworten, d.h. eine Kalzium- 


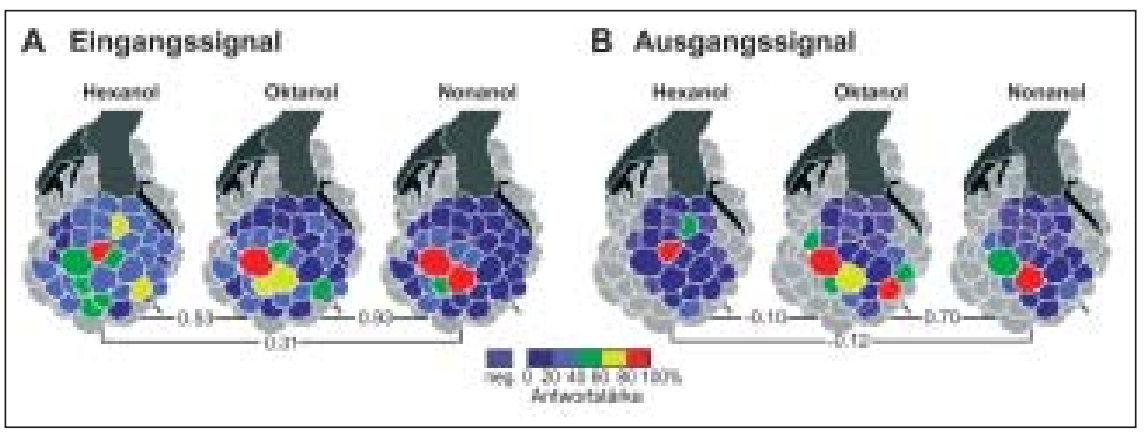

Abb. 3: Das Ausgangssignal ist gegenüber dem Eingangssignal kontrastverstärkt. (A und B) Die räumlichen Aktivitätsmuster verschiedener Düfte sind in einem standardisierten AL dargestellt. Die Muster wurden über verschiedene Tiere gemittelt. Die Antwortstärke wurde in fünf Kategorien eingeteilt, inhibitorische Antworten sind in einer weiteren Kategorie repräsentiert (neg.). Graue Glomeruli konnten nicht gemessen und somit nicht charakterisiert werden. Die Korrelationen als Maß der Ähnlichkeit der verschiedenen Duftpaare sind unterhalb der ALs dargestellt.

abnahme während der Duftgabe, sind in einer weiteren Kategorie repräsentiert, die nur auf der Ausgangsebene vorkommt, wodurch die Kodierungsmöglichkeiten erweitert werden. Ein Vergleich des Eingangs- mit dem Ausgangssignal zeigt, wie schon aus Abbildung 2 ersichtlich, dass sich die räumlichen Muster der drei Düfte ähnlich sind. Hexanol wird durch einen zentralen Glomerulus dominiert (Glomerulus 28), Oktanol und Nonanol aktivieren jeweils Glomeruli 17 und 33 am stärksten. Vergleicht man nun die Antwortstärken der mittelstark aktivierten Glomeruli, so erhalten diese zwar einen Rezeptorneuroneingang, ihre Aktivität ist aber im Ausgangssignal stark reduziert. Glomerulus 17 zum Beispiel erhält während der Hexanolstimulation ein mittleres Eingangssignal, welches jedoch im Ausgang nicht sichtbar

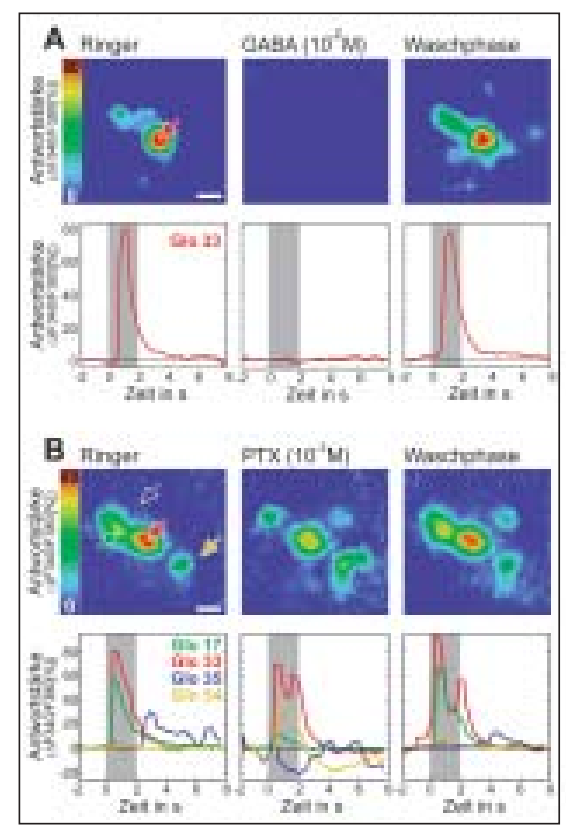

lokalen Interneuronen abgegeben und spielt im AL der Honigbiene eine prominente Rolle (Schäfer und Bicker 1986). Wir haben deshalb den Einfluss der GABA ergen Interneurone auf die duftspezifischen Aktivitätsmuster der Projektionsneurone untersucht. Hier$\mathrm{zu}$ haben wir GABA und den Antagonisten Picrotoxin (PTX) appliziert und die Veränderungen der Duftantworten analysiert (Sachse und Galizia 2002b). In Abbildung 4 sind die räumlichen und zeitlichen Duftantworten auf Nonanol vor, während und nach der GABAbzw. PTX-Gabe dargestellt. GABA bewirkte eine komplette Reduktion der Aktivitätsmuster der Projektionsneurone. Der Effekt war reversibel und die Kalziumsignale kehrten in der Auswaschphase wieder zurück. Dieses Ergebnis zeigt, dass das Netzwerk GABA-sensitiv ist und durch diesen Neurotransmitter inhibiert wird. PTX hingegen blockierte die inhibitorische GABA-Transmission und rief somit gegensätzliche und sehr komplexe Effekte hervor. Generell bewirkte PTX eine signifikante Erhöhung der intrazellulären Kalziumkonzentration in allen Glomeruli. Im speziellen veränderte PTX die Antworteigenschaften spezifischer Glomeruli während der Duftgabe und veränderte damit das duftspezifische Aktivitätsmuster. Da PTX inhibitorische Verschaltungen im AL blockiert, erstaunt es nicht, dass die Antwortstärke einiger Glomeruli zunahm und sich die Anzahl aktiver Glomeruli erhöhte (Glomeruli 33 und 54 in Abbildung 4B). Interessanterweise aber blieben Glomeruli, die eine inhibitorische Duftantwort gezeigt haben, auch während der PTX-Gabe inhibitorisch (z.B. Glomerulus 35). Zudem wurde die Antwortstärke einiger Glomeruli stark reduziert (z.B. Glomerulus 17). Es muss also ein weiteres inhibitorisches Netzwerk im AL vorhanden sein, das PTXunempfindlich ist.

Ein Vergleich der Duftantworten der Glomeruli vor und während der PTX-Behandlung gibt Aufschluss über das inhibitorische Netzwerk innerhalb des ALs. In Abbildung 5A sind schematisch die Antworten dreier Glomeruli (a bis c) vor und während PTXGabe dargestellt, welche den Glomeruli 54, 33 und 35 aus Abbildung 4B entsprechen. In Abbildung 5B ist ein mögliches Verschaltungsmodell für diese drei Glomeruli gezeigt, anhand dessen man die beobachteten PTX-Effekte erklären kann. Jeder Glomerulus in diesem Modell besitzt einen Rezeptorneuron-Eingang und einen Projektionsneuron-Ausgang. Die Glomeruli sind in ein globales inhibitorisches Netzwerk involviert (rote Verbindungen), welches PTX-sensitiv ist. Dieses Netzwerk kontrolliert die globale Aktivität im AL, wie etwa bei einer Pupil- 


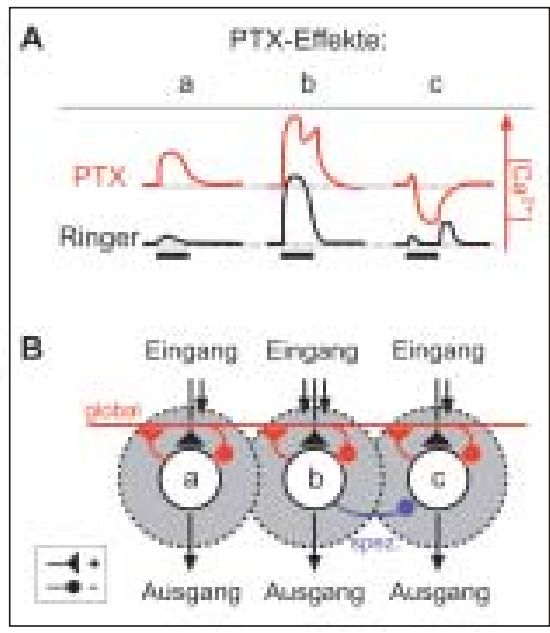

Abb. 5: (A) Schematische Darstellung der PTX-Effekte für drei Glomeruli. Die schwarzen Zeitverläufe entsprechen den Glomeruliantworten vor der PTX-Behandlung; die roten Zeitverläufe stellen die Antworten während PTX dar. (B) Verschaltungsmodell des ALs, welches auf den PTX-Ergebnissen basiert. Die drei Glomeruli (a bis c) stehen für die drei schematisierten PTX-Effekte in A. Jeder Glomerulus erhält einen Rezeptorneuron-Eingang. Die Duftinformation wird im AL durch zwei inhibitorische Netzwerke verarbeitet. Ein globales PTX-sensitives Netzwerk (rot) und ein glomerulus-spezifisches PTX-insensitives Netzwerk (blau). Über den Projektionsneuron-Ausgang wird die modulierte und kontrastverstärkte Duftinformation an höhere Verarbeitungsstrukturen gesendet.

le, die sich bei starkem Licht schließt und dadurch alles Licht in der Stärke reduziert. Das zweite, PTX-insensitive Netzwerk ist nicht global, sondern bewirkt spezifische Kontraste zwischen individuellen Glomeruli. Wenn ein stark aktivierter Glomerulus einen schwächer aktivierten Glomerulus spezifisch inhibiert (blaue Verschaltung), wird die relativ unscharfe Rezeptorantwort kontrastverstärkt. Solch eine inhibitorische Verschaltung ist von Glomerulus b auf Glomerulus c gezeigt, was dazu führt, dass dieser Glomerulus ein inhibitorisches Ausgangssignal besitzt, obwohl er einen RezeptorneuronEingang bekommt. Wenn man nun PTX zum AL appliziert, blockiert man das globale inhibitorische Netzwerk (die roten Verschaltungen fallen weg). Dadurch erhöht sich die Antwort der stark und mittelstark aktivierten Glomeruli (Glomeruli a und b), während die inhibitorische Antwort von Glomerulus c erhalten bleibt, da diese auf dem spezifischen PTX-insensitiven Netzwerk beruht. Die durch PTX hervorgerufene Reduktion von Duftantworten (z.B. Glomerulus 17 in
Abbildung 4B) ist bisher ungeklärt und kann nicht anhand dieser zwei Netzwerke erklärt werden. Zusätzlich ist auch der Transmitter des spezifischen Netzwerkes unbekannt. Dieses Modell stellt also nur einen Teil des AL Netzwerkes dar. Die wirklichen Verschaltungen sind wahrscheinlich wesentlich komplizierter und erfordern weiterführende Experimente.

Zusammengefasst zeigen diese Studien, dass komplexe Verschaltungen im AL, basierend auf mindestens zwei inhibitorischen Netzwerken, die überlappenden Antwortspektren der Rezeptorneurone verschärfen und optimieren. Diese Verarbeitung führt zum einen dazu, dass die Repräsentationen von ähnlichen Düften auf der AL-Ausgangsebene unterscheidbarer werden. Zum anderen bewirkt diese Duftverarbeitung im AL eine konzentrationsinvariante Repräsentation eines Duftes über mehrere Konzentrationsstufen und liefert somit die Grundlage dafür, dass eine Biene einen Duft auch bei unterschiedlichen Konzentrationen auf einer Blütenwiese wiederfindet (Sachse und Galizia 2002a). Kürzlich haben wir auch die Verarbeitung von Duftmischungen im AL analysiert, da die meisten vorkommenden Düfte als komplexe Mischungen auftreten. Die Ergebnisse zeigen, dass Duftmischungen nicht als eine einfache Summe der Einzeldüfte kodiert werden, sondern die stark aktivierten Glomeruli eine reduzierte Aktivität aufweisen, welche auf den inhibitorischen Verschaltungen im AL beruht (Sachse und Galizia, unveröffentlicht). Dieser Mechanismus könnte eine ausreichende Kodierungskapazität für komplexe Mischungen gewährleisten. Diese drei Beispiele zeigen, dass schon auf der Ebene der ersten olfaktorischen Schaltstation im Gehirn ein beträchtlicher Anteil an der Duftverarbeitung stattfindet, der die Grundlage für das leistungsstarke Geruchssystem der Honigbiene liefert.

\section{Danksagung}

Wir danken Randolf Menzel für seine uneingeschränkte Unterstützung und für wertvolle Diskussionen, Philipp Peele für nützliche Kommentare und Heinz Busert für das Bienenbild.

\section{Literatur}

Abel, R., Rybak, J. und Menzel, R. (2001): Structure and response patterns of olfactory interneurons in the honeybee. Apis mellifera. J. Comp. Neurol. 437: 363-383.

Arnold, G., Masson, C. und Budharugsa, S. (1985): Comparative study of the antennal lobe and their afferent pathway in the worker bee and the dro- ne (Apis mellifera). Cell Tissue Res. 242: 593605.

Bicker, G., Kreissl, S. und Hofbauer, A. (1993): Monoclonal antibody labels olfactory and visual pathways in Drosophila and Apis brains. J. Comp. Neurol. 335: 413-424.

Esslen, J. und Kaissling, K.-E. (1976): Zahl und Verteilung antennaler Sensillen bei der Honigbiene (Apis mellifera L.). Zoomorphol. 83: 227 251.

Faber, T., Joerges, J. und Menzel, R. (1999): Associative learning modifies neural representations of odors in the insect brain. Nat. Neurosci. 2: 74-78.

Flanagan, D. und Mercer, A.R. (1989): An atlas and 3-D reconstruction of the antennal lobe in the worker honeybee, Apis mellifera L. (Hymenoptera: Apidae). J. Insect. Morphol. Embryol. 18: 145-159.

Frisch, K.v. (1993): Aus dem Leben der Bienen Berlin-Göttingen-Heidelberg: Springer-Verlag.

Galizia, C.G., McIllwrath, S.L. und Menzel, R. (1999a): A digital three-dimensional atlas of the honeybee antennal lobe based on optical sections acquired by confocal microscopy. Cell Tissue Res. 295: 383-394.

Galizia, C.G. und Menzel, R. (2001): The role of glomeruli in the neural representation of odours: results from optical recording studies. J. Insect Physiol. 47: 115-130.

Galizia, C.G., Sachse, S., Rappert, A. und Menzel, R. (1999b): The glomerular code for odor representation is species specific in the honeybee Apis mellifera. Nat. Neurosci. 2: 473-478.

Hammer, M. (1997): The neural basis of associative reward learning in honeybees. Trends $\mathrm{Neu}$ rosci. 20: 245-252.

Hildebrand, J.G. und Shepherd, G.M. (1997): Mechanisms of olfactory discrimination: Converging evidence for common principles across phyla. Annu. Rev. Neurosci. 20: 595-631.

Joerges, J., Küttner, A., Galizia, C.G. und Menzel, R. (1997): Representation of odours and odour mixtures visualized in the honeybee brain. $\mathrm{Na}$ ture 387: 285-288.

Mobbs, P.G. (1982): The brain of the honeybee Apis mellifera. I. The connections and spatial organization of the mushroom bodies. Philos. Trans. R. Soc. London B Biol. Sci. 298: 309-354.

Mombaerts, P. (1999): Seven-transmembrane proteins as odorant and chemosensory receptors. Science 286: 707-711.

Mori, K. und Yoshihara, Y. (1995): Molecular recognition and olfactory processing in the mammalian olfactory system. Prog. Neurobiol. 45: 585-619.

Müller, D., Abel, R., Brandt, R., Zockler, M. und Menzel, R. (2002): Differential parallel processing of olfactory information in the honeybee, Apis mellifera L. J. Comp. Physiol. A 188: 359370.

Sachse, S. und Galizia, C.G. (2002a): Intensity-invariance of odor-quality is optimized by the antennal lobe network in the honeybee Apis mellifera. 32nd Annual Meeting SfN, Orlando, USA, Poster No. 561.19.

Sachse, S. und Galizia, C.G. (2002b): Role of inhibition for temporal and spatial odor repre- 
sentation in olfactory output neurons: a calcium imaging study. J. Neurophysiol. 87: 1106-1117.

Sachse, S., Rappert, A. und Galizia, C.G. (1999):

The spatial representation of chemical structures in the antennal lobes of honeybees: steps towards the olfactory code. Eur. J. Neurosci. 11: 39703982.

Schäfer, S. und Bicker, G. (1986): Distribution of GABA-like immunoreactivity in the brain of the honeybee. J. Comp. Neurol. 246: 287-300.

Sicard, G. und Holley, A. (1984): Receptor cell responses to odorants: Similarities and differences among odorants. Brain Res. 292: 283-296.

Suzuki, H. (1975): Antennal movements induced by odor and central projection of the antennal neurones in the honeybee. J. Insect Physiol. 21: 831-847.

Vosshall, L.B. (2000): Olfaction in Drosophila. Curr. Opin. Neurobiol. 10: 498-503.

Witthöft, W. (1967): Absolute Anzahl und Verteilung der Zellen im Hirn der Honigbiene. $Z$. Morph. Tiere 61: 160-184.

Young, J.M. und Trask, B.J. (2002): The sense of smell: genomics of vertebrate odorant receptors. Hum. Mol. Genet. 11: 1153-1160.

\section{Kurzbiographien der Autoren}

Silke Sachse, Studium der Biologie an der Freien Universität Berlin. 2002 Promotion an der FU Berlin. Seit Oktober 2002 Postdoc an der Rockefeller Universität in New York im Labor von Asst. Prof. Dr. Leslie Vosshall.

C. Giovanni Galizia, Studium der Biologie an der Freien Universität Berlin. 1993 Promotion an der University of Cambridge in England. 1993 Postdoc am MPI für Entwicklungsbiologie in Tübingen, 1995 Postdoc an der FU Berlin. Seit 1999 Leiter einer von der Volkswagenstiftung eingerichteten Nachwuchsgruppe an der FU Berlin (2002 Habilitation), seit 2003 Associate Professor an der University of California, Riverside.

\section{Korrespondenzadressen}

\section{Silke Sachse}

The Rockefeller University, Laboratory of Neurogenetics and Behavior

1230 York Avenue, Box 63

New York, NY 10021/USA

Tel.: ++1-212-327 7239

Fax: $++1-212-3277238$

e-mail:sachses@mail.rockefeller.edu

\section{Giovanni Galizia}

Department of Entomology

University of California, Riverside

Riverside, CA 92506 USA

Tel.: ++1909827 7051

Fax: ++19097873086

e-mail: galizia@ucredu 